\title{
Probing Nucleon Structure with Neutrinos
}

\author{
D.H. Perkins* \\ Nuclear Physics Laboratory, University of Oxford, \\ Keble Road, Oxford OX1 3RH, UK \\ E-mail: id.perkins1@physics oxford.ac.uki
}

Abstract: Talk given on receiving the "High Energy and Particle Physics Prize" of the

European Physical Society.

It is a great honour and privilege to be here in Budapest to receive this award from the European Physical Society. First of all, however, I must emphasize that the work it refers to was a joint effort by many people, not just particle physicists but also engineers, beam and chamber specialists, accelerator experts, teams of film scanners and so on. So I am really accepting this award on behalf of, and as representative of, all the many people with whom I was privileged to work over the years.

The story of neutrinos and quark structure goes back a very long way, to before many of you were born, in fact to 38 years ago, in 1962/3, when for the first time a neutrino beam from the CERN PS produced interactions in a bubble chamber, the CERN $1.2 \mathrm{~m}$ heavy liquid chamber, holding around one ton of freon. The results which I gave at the Sienna Conference in 1963 and which were later published showed that the inelastic neutrino crosssection on nucleons in the region of a few $\mathrm{GeV}$ was large - almost an order of magnitude bigger than the elastic cross-section - and moreover increasing rapidly with energy (see Figure 1). They were clearly trying to tell us a very simple thing, but unfortunately, we were just not listening!

It was to take another 5 years before it became clear, to me at least, what that distribution meant. Then, at the 1968 Vienna Conference, Panofsky presented the first data on deep inelastic electron scattering on protons from the SLAC- MIT experiment of Friedman, Kendall and Taylor. Panofsky was very careful and cautious in his claims. He talked about the interpretation in terms of "Bjorken scaling" but, as I recall, in a one hour talk, only mentioned the words "pointlike structure" once. But as soon as he showed Henry Kendall's curves (Figure 2) of $W_{2}$ against energy transfer $\nu$ for various values of $q^{2}$ between 0.8 and $2.2 \mathrm{GeV}^{2}$, and how they ran together at high energies, the bells rang out for me loud and clear. It was so simple! If at high energy transfer, $W_{2}$ was really independent of $q^{2}$, then neutrinos, assuming they were probing the same structure in the

\footnotetext{
${ }^{*}$ Speaker.
} 


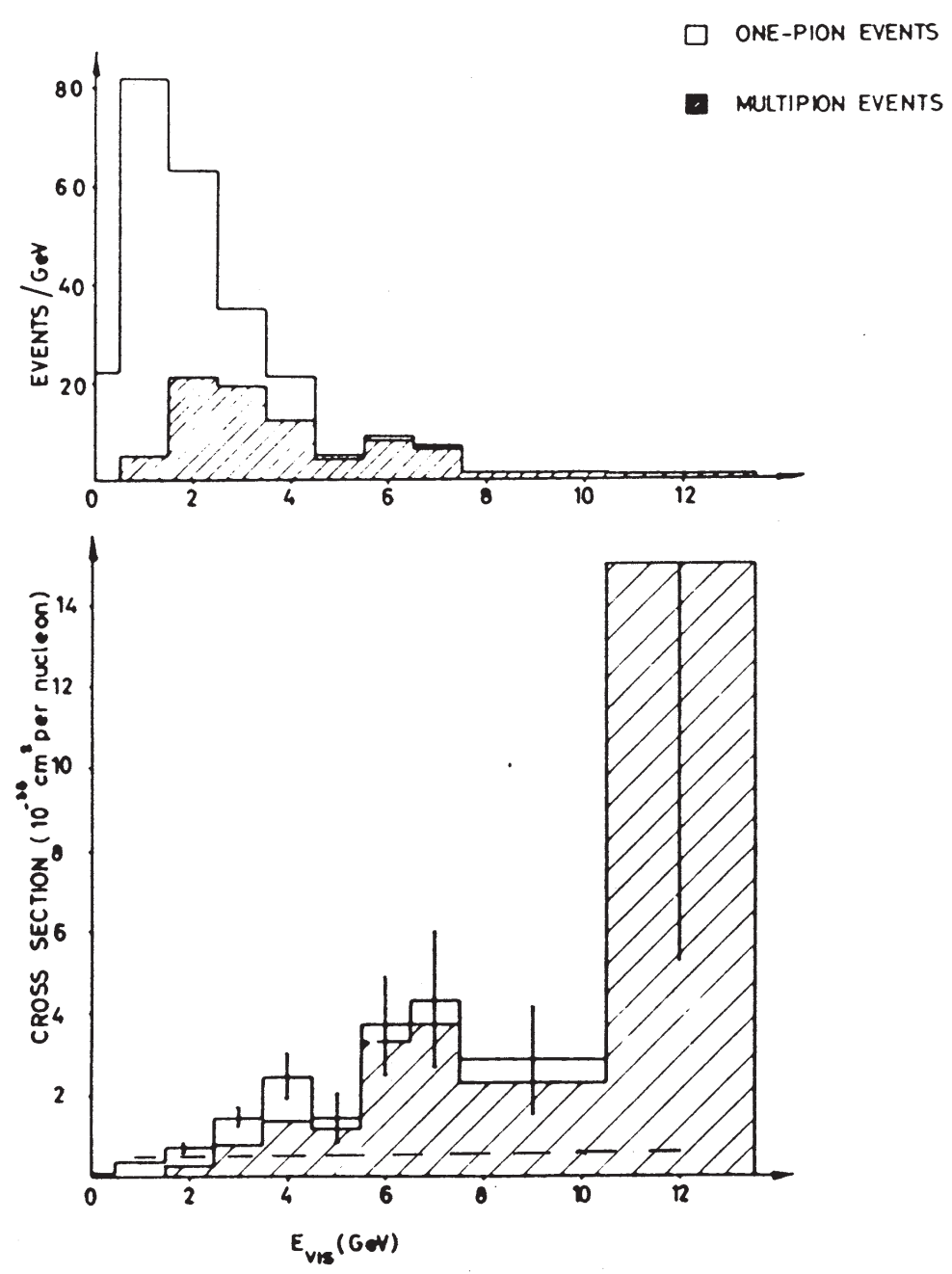

Figure 1: Early (1963) CERN results on inelastic neutrino event rates (top) and cross-section (bottom) as function of estimated neutrino energy. The dashed line shows the value of the quasielastic cross section.

nucleon, should have total cross-sections proportional to $q^{2}(\max )$, or rising linearly with laboratory neutrino energy. Going back to CERN, I re-plotted the old freon data and the more recent propane data. It was indeed linear with neutrino energy (see Figure 3). This just shows you that, when you know what the answer is, it's easy! Hindsight is indeed wonderful. Since the pointlike behaviour was only expected for large energy transfers in the deep inelastic region, it was remarkable that the energy dependence was linear to quite low energies. In the jargon of the time, it was called "precocious scaling".

However, the proof that these pointlike constituents, behaving in a peculiar way as if almost free particles, were the long-sought quarks, had to await another five years, and the arrival in CERN of the large French heavy liquid chamber Gargamelle, conceived, designed and built by André Lagarrigue at Saclay. With 10 times the sensitive mass of the old CERN chamber, and an enhanced beam, we obtained a few thousand events instead of a 


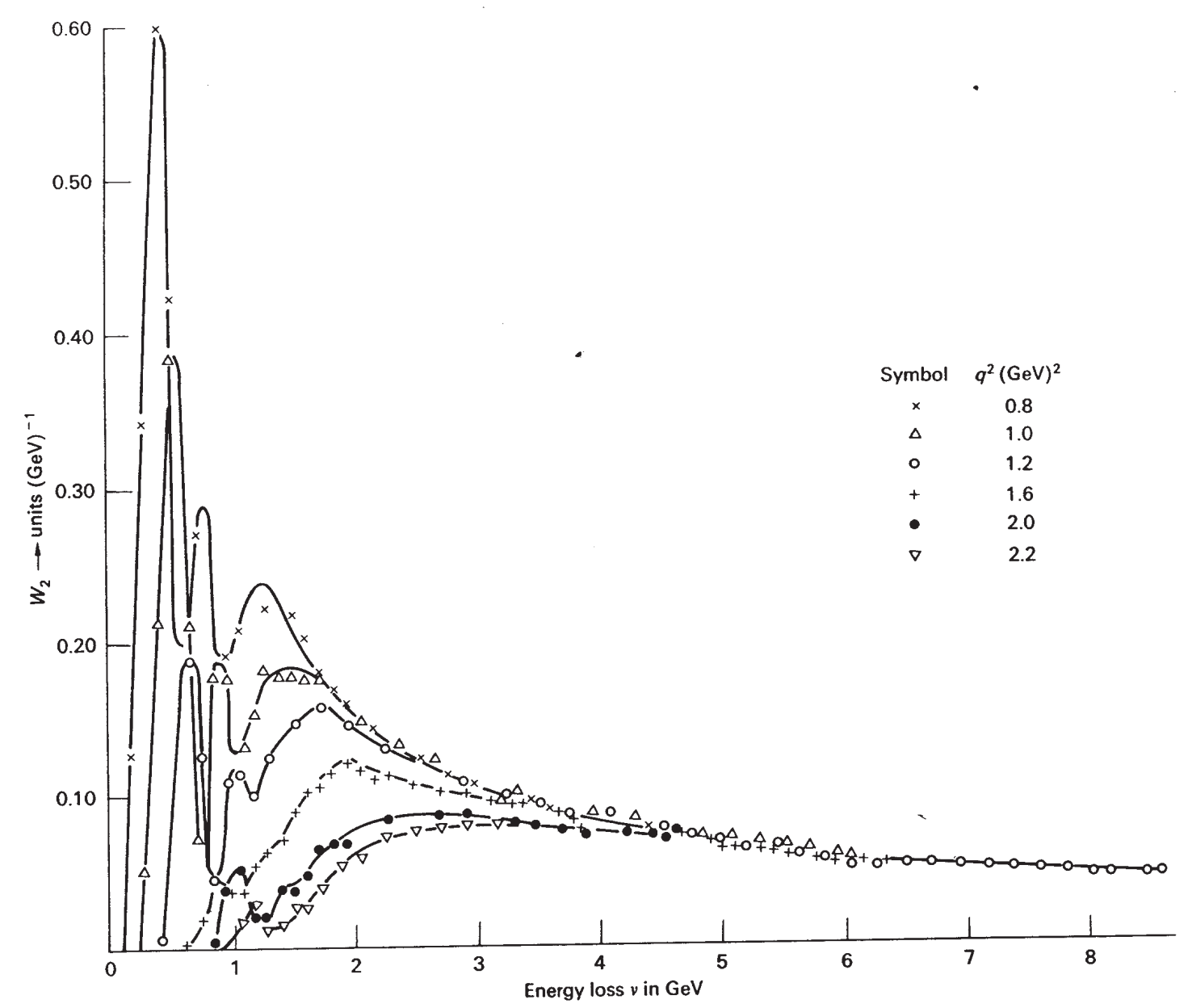

Figure 2: Plot of the first SLAC-MIT data on deep inelastic ep scattering shown by Panofsky (Vienna 1968). The structure function $W_{2}\left(=F_{2} / \nu\right.$ in modern parlance) is plotted against energy transfer $\nu$ for values of $q^{2}$ from 0.8 to $2.2 \mathrm{GeV} / \mathrm{c}^{2}$. The very weak dependence of $W_{2}$ on $q^{2}$ at large $\nu$ was the smoking gun of the SLAC-MIT data.

hundred or so. Most importantly, there was antineutrino as well as neutrino data. The total charged-current cross-sections in both cases were linear with energy (see Figure 4), with the antineutrino cross-section equal to just over one third of the neutrino cross- section, thus confirming the earlier Stanford result that the pointlike constituents had half-integral spin. From the two sets of data, one could extract the structure function $F_{2}(x)$ and compare it directly with the electron scattering data on nucleons (deuterons). Of course SLAC had millions of events and Gargamelle only 3000, but it was clear that both were seeing the same structure, and they agreed in absolute magnitude to within $10 \%$ accuracy if the SLAC data points were divided by $5 / 18$, the mean square charge of the $u$ and $d$ quarks in the nucleon according to Gell-Mann and Zweig (see Figure 5). Of course the two experiments were very different: carried out in different laboratories, using different probes, different fundamental interactions and totally different detector technology. I remember remarking 

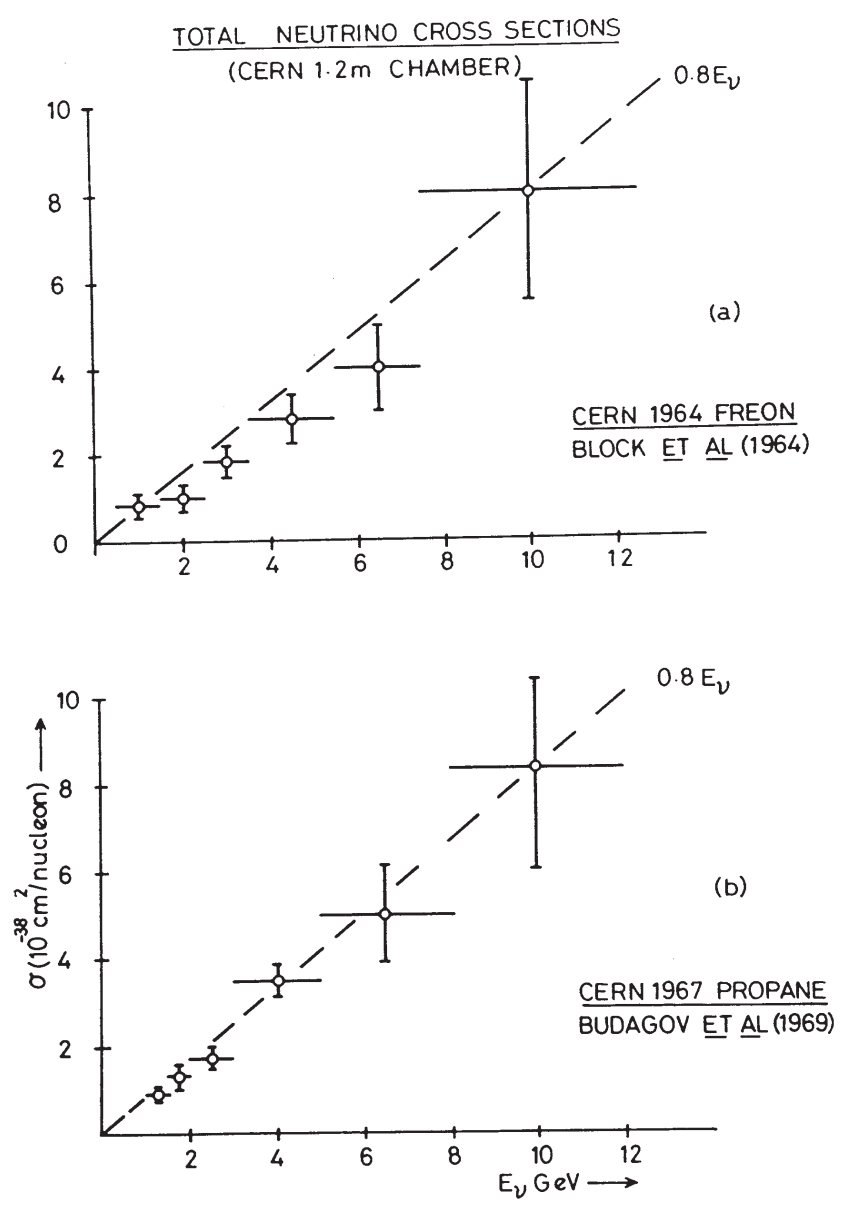

Figure 3: Plots of total neutrino cross-sections from the old (1963) freon data and the more recent (1967) propane data, consistent with the linear energy dependence expected from the plot of Figure 2 .

at the Fermilab Conference in 1972 that the agreement seemed somewhat miraculous! Well, miracles do happen.

The last stage in the saga of the probing of quarks with neutrino beams and bubble chambers was to take yet another five years. In 1978, the structure functions measured at low $q^{2}$ in Gargamelle with the PS beam could be compared with those measured at higher $q^{2}$ with the BEBC chamber, filled with heavy liquid (neon-hydrogen) and running in a high energy neutrino beam from the CERN SPS. For the non-singlet structure function $F_{3}$, obtained from the neutrino- antineutrino cross-section difference, perturbative QCD made a very simple prediction. Taking moments and integrating both sides of the Altarelli-Parisi evolution equation, there was a neat factorization with the result that the moment was predicted to vary as $\log q^{2}$ to a certain power, called the anomalous dimension. So if two different moments, say $n=3$ and $n=5$, were plotted against each other as a function of $q^{2}$ on a log-log scale, one should get a straight line with a slope equal to the known ratio of anomalous dimensions. Amazingly, the observed slopes (Figure 6) agreed almost 


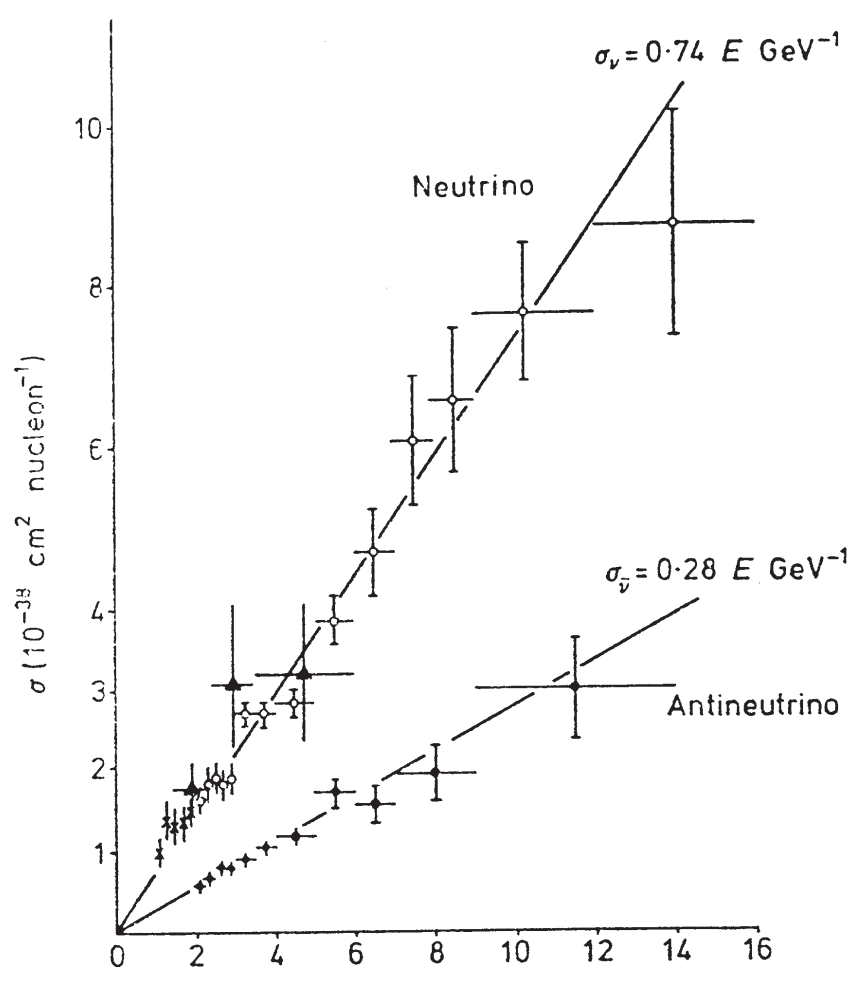

Figure 4: Neutrino and antineutrino charged current cross-sections measured in Gargamelle with the PS neutrino beam. Both are proportional to energy and the antineutrino/neutrino cross-section ratio of about $1 / 3$ indicates spin $1 / 2$ parton constituents.

exactly with theory. I believe this was the first quantitative test of QCD. I must say that, when I first plotted these moments, and saw how remarkably the QCD predictions fitted them, my admiration and respect for theoretical physicists shot up by leaps and bounds (starting however from a rather low level). Other possible models, for example fixed point theories, would not predict any particular slopes, while scalar gluons predicted much flatter slopes and were excluded at the 4 standard deviation level. Our data was hardly in the perturbative domain, so I think we were just lucky. Perhaps, after 15 years on the job, we deserved a bit of luck.

Of course, during this long saga, we had many bad days and a few good ones, and some memorable moments. I felt I could not give an address about the experimental verification of the nature of partons without at least one story about Richard Feynman, the inventor of the parton model. At a meeting in Hawaii in 1973, Feynman gave a number of lectures on the subject.( I should say here that, at this meeting, the quark-parton model was for me something of a side issue: my main task there was to present the experimental discovery of neutral weak currents in the Gargamelle chamber. To convince Feynman - initially a total disbeliever - of those results involved me in one of the biggest scientific fights of my life). Anyhow, one of the people at the meeting was the theorist Chris Llewellyn-Smith. He found out that I had all the Gargamelle kinematics output with me, and asked if I could 


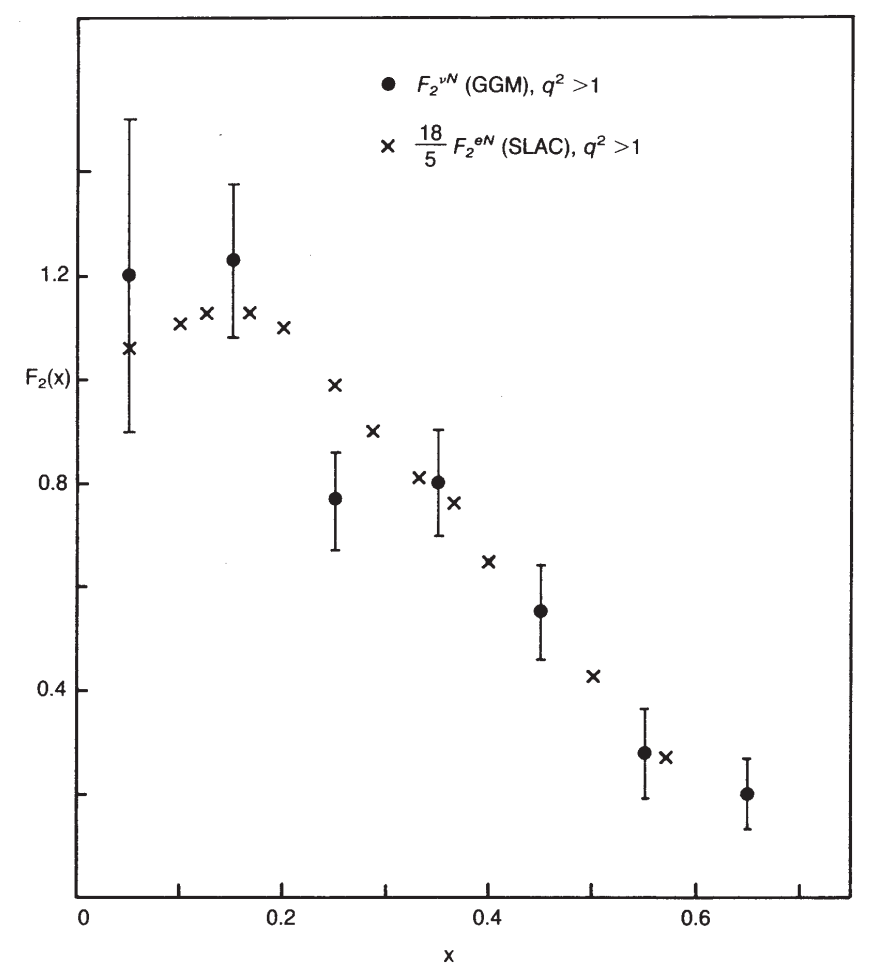

Figure 5: $F_{2}(x)$ per nucleon, as measured from SLAC deep inelastic electron- deuteron scattering (crosses) and from Gargamelle neutrino/antineutrino data. The SLAC-MIT values have been divided by $5 / 18$, the mean square charge of the $u$ and $d$ quarks in the nucleon according to Gell-Mann and Zweig.

check the sumrule that he and David Gross had come up with: that the integral of $F_{3}$ from $x=0$ to 1 was equal to the number (three) of valence quarks in the nucleon. So while Chris was sunning himself on the beach at Waikiki, I worked on his sumrule. Next morning he asked if I had a result. I told him the integral came out somewhere between 1 and 4 , the result being uncertain because of large statistical errors, on whether one integrated over Bjorken $x$ or the Bloom-Gilman variable $x^{\prime}$ favoured by SLAC, and on how I extrapolated the data to $x=0$ to complete the integral. And of course the values of $q^{2}$ were small and not the infinite values required by the sumrule (this was before the $\alpha_{s}$ corrections at finite $q^{2}$ were known about). My best estimate was $2.5 \pm 1$, but that was not an official Gargamelle number and just for his private information.

The first lecture that day was by Feynman and by sheer coincidence he mentioned the GLS sumrule, and that his friend Barish at CalTech would very soon have this number from a neutrino experiment which was just starting at Fermilab. This, said Feynman, would be the final acid test of the quark model, and we must just wait for Barish's result. LlewellynSmith interjected that as of today, the number had already been measured. Feynman, somewhat surprised, of course asked what it was, and Chris told him. It is the only time I saw Feynman stopped dead in his tracks - for at least 2 seconds! Then he turned round and said just two words: HOT DOG! 


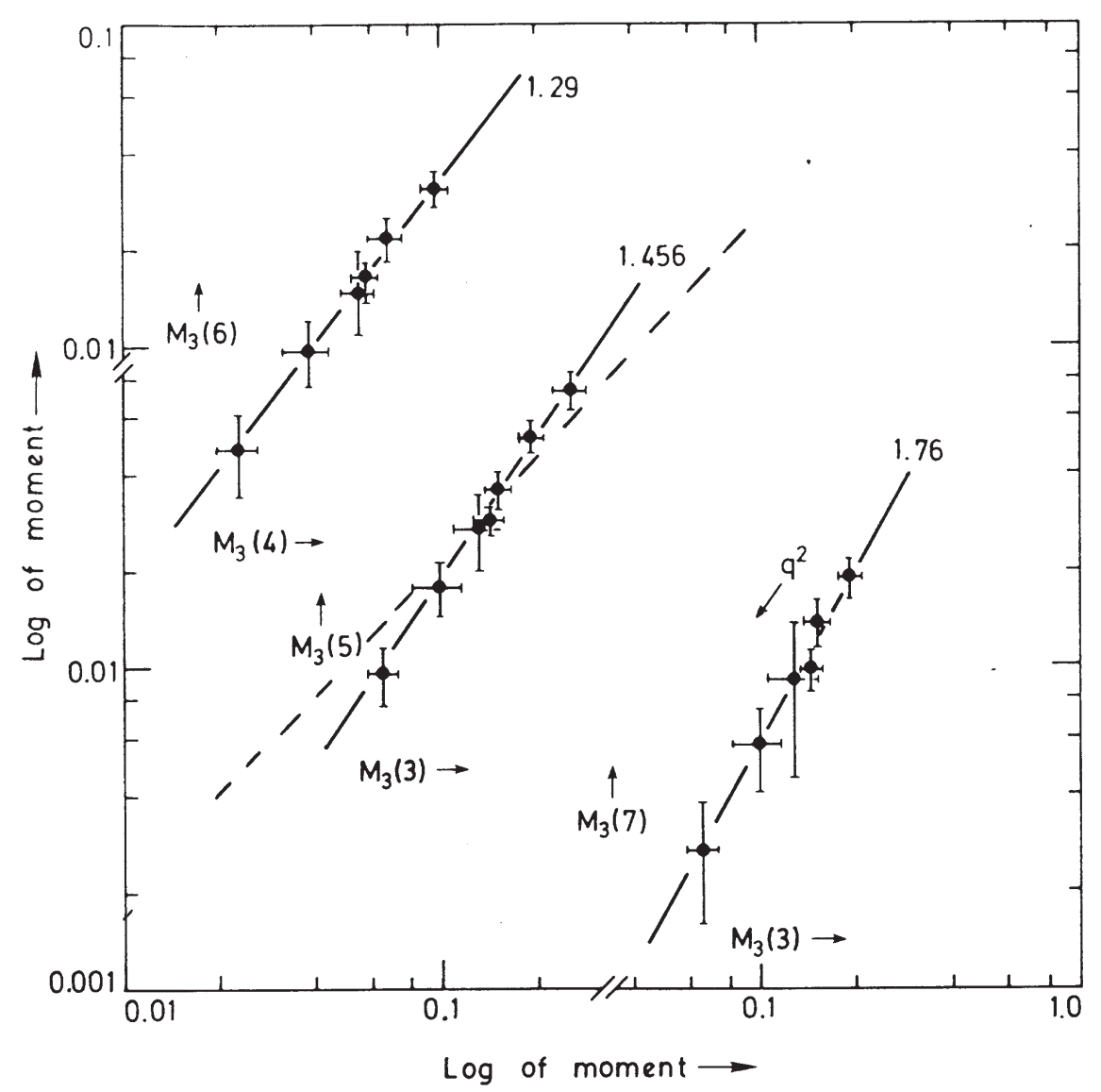

Figure 6: Plots of moments of the non-singlet structure function $F_{3}\left(x, q^{2}\right)$, from the Gargamelle and BEBC data. The moments are the Nachtmann moments which include mass corrections. In each case, the logarithm of one moment (say $n=5$ ) is plotted against another (say $n=3$ ) as $q^{2}$ varies over the range $5-50 \mathrm{GeV} / \mathrm{c}^{2}$. Perturbative QCD predicts a linear relation with the slopes indicated. The dashed line for $n=5$ and $n=3$ is for scalar gluons. 